\title{
BREAST CANCER IN YOUNG PATIENTS: PROGNOSTIC AND PROFILE EPIDEMIOLOGICAL ANALYSIS IN A TERTIARY HOSPITAL
}

Amilcar Alves Assis¹, Mauro Passos¹, Rodrigo Kouzak¹, Karoline Evangelista1', Natasha Caldas¹

${ }^{1}$ Hospital de Base do Distrito Federal - Brasília (DF), Brazil.

Breast cancer is the second most prevalent and first in mortality in Brazilian women. Its incidence has increased in recent years in all age groups. According to the Instituto Nacional do Câncer in 2019, 59,700 new cases of breast cancer are expected, with an estimated risk of 56.33 cases per 100 women. The diagnosis of breast cancer is more frequent in women after 50 years of age; it is estimated that only $25 \%$ of all cases occur in women below the age of 50 years; however, there was a literature consensus that tumors in this young age group have a worse prognosis, both because they are biologically more aggressive and because of affect women outside the screening age group in Brazil; thus, the rate of locally advanced disease at diagnosis in this age group is considerably higher. It is suggested that early onset breast cancer is related to different etiological factors, histopathological aspects, and clinical outcomes, as compared to postmenopausal breast cancer. Thus, age becomes an important prognostic factor. Since breast cancer is a curable pathology, the type of therapeutic approach also varies, with proposed treatment tends to be more aggressive. With the advent and increasing availability of genetic tests, predisposition of breast cancer has increased the number of indications for prophylactic mastectomies, especially in younger age groups or notably in patients with known pathological mutations in BRCA1 and BRCA2 genes. However, the literature is still controversial regarding its impact on overall survival. Breast cancer diagnosed before the age of 50 years is a behavioral disease, with prognosis and approach very different from that diagnosed in postmenopausal women. Therefore, it is important to know the profile of these patients to provide optimal treatment and achieve the best outcomes.

Keywords: Breast Cancer; Young Women; Premenopause. 